\title{
Spitalentwicklung geht nur mit den Ärztinnen und Ärzten
}

Gaby Belz

Interessenverbindungen: Die Autorin ist Inhaberin eines Unternehmens für Organisationsentwicklung, das auch Institutionen und Organisationen des Gesundheitswesens berät.

Korrespondenz: Gaby Belz Obere Felsenstrasse 7 CH-9000 St. Gallen Tel. 0719512415

gaby[at]belz-gmbh.ch

\section{Mehrfacher Druck auf Spitäler}

Gesundheit ersetzt heute Religion. Entsprechend hoch sind die Erwartungen von Patientinnen und Patienten an die Fachkräfte im Spital. Der Zugang via Internet zu nicht validierten Informationen führt zu einem nie dagewesenen Wissensstand bei den Betroffenen. Gleichzeitig ist wenig Bewusstsein vorhanden für die komplexen Wechselwirkungen und die vielen atypischen Verläufe, die eindeutige diagnostische Aussagen und klare Behandlungswege erschweren können. Medizinerinnen sowie Angehörige anderer Disziplinen sind heute fachlich wie kommunikativ stark gefordert.

Der zweite Brennpunkt ist die Gesundheitspolitik mit dem Ziel der Kostensenkung bei guter (gut genug?) Diagnose- und Behandlungsqualität. Einzelne Häuser werden geschlossen, andere wurden in profitorientierte Trägerschaftsformen überführt und auf betriebswirtschaftliche Maximen umgestellt. Die Schwerpunktbildung in der Spitzenmedizin löst einen nie dagewesenen Wettbewerb zwischen den grossen Spitälern aus. Die spitalinternen Widerstände gegen die Schaffung aussagekräftiger Informationsgrundlagen zu den betriebswirtschaftlichen Aspekten der Unternehmensführung erschweren es, zu klaren Daten zu kommen. Der hohe Organisationsgrad der Gesundheitsversorgungs-Lobby verhindert überdies die Durchsetzung einer zügigen, zukunftsorientierten und qualitätsbewussten Strategie. Das Wissen dafür wäre - mit den erwähnten Einschränkungen - längst vorhanden.

Schliesslich sind in den zwei tragenden Berufsgruppen - Medizin, Pflege - ausgetrocknete Arbeitsmärkte zu verzeichnen. Das erschwert eine echte Selektion bei der Rekrutierung und verursacht Belastungen in den Teams. Qualitäts- und Zusammenarbeitsprobleme können sich in Form von hohen Fluktuationsraten und überdurchschnittlichen Krankheitsausfällen auch bei den Personalkosten niederschlagen. Die damit verbundenen menschlichen Belastungen auf allen Stufen kann man nicht quantifizieren, sie sind aber ein Faktor.

In grossen Häusern wurden die Führungsspannen optimiert. Damit können Führungskräfte auf Departements- bzw. Bereichs-Ebene erstmals gestaltend eingreifen. Diese Anpassung entspricht dem Usus in Wirtschaft und Verwaltung. Die bisher üblichen, sehr grossen Entscheidspielräume auf Stufe Chefarzt/-ärztin werden aber damit beschnitten, und der direkte Zugang zur Spitaldirektion ist durch eine zusätzliche Stufe so nicht mehr möglich. Wider-

\section{Les hôpitaux ne peuvent}

\section{se développer qu'en collaboration avec les médecins}

Les cadres dirigeants sont sous pression: attentes élevées des patients envers les médecins et les soignants, pression sur les coûts, concurrence accrue entre hôpitaux, charge administrative accrue due à l'obligation de se justifier envers les assureurs, assèchement du marché du travail dans le secteur de la médecine et des soins.

L'idée que de nombreux médecins dirigeants se font de leur profession tient trop peu compte des concepts de gestion d'entreprise intégratifs et interdisciplinaires. Au vu des énormes exigences auxquelles les médecins-chefs sont confrontés, ceux-ci exigent en contrepartie une grande liberté d'action. Des améliorations substantielles ne peuvent être obtenues que si les médecins y voient un avantage.

Nos propositions:

- Gestion flexible de la fonction dirigeante: temps partiel, partage de poste, carrières parallèles pour les responsables et les gestionnaires, gestion partagée entre médecins et soignants.

- Culture d'entreprise et de gestion axée sur la réalisation interprofessionnelle du mandat de prestations, échange régulier d'expériences entre dirigeants («learning community»), instauration d'une réelle communication interprofessionnelle à tous les niveaux.

- Mise en place d'offres stratégiques de développement personnel pour devenir un employeur attractif, engager et garder les meilleurs, et pousser les bons à devenir meilleurs.

- Echange durable avec les principaux groupes partenaires pour un solide ancrage d'une culture d'apprentissage et de développement dans l'établissement.

La direction de I'hôpital doit montrer l'exemple et faire preuve d'un esprit d'équipe irréprochable. 
stände sind vielerorts nicht $\mathrm{zu}$ vermeiden. Dass damit auch eine substantielle Entlastung für die Führungspositionen verbunden ist, wurde noch nicht überall erkannt und verstanden. Spitaldirektionsposten sind heute besetzt mit Persönlichkeiten, die einen ausgeprägten Führungsanspruch mit modernen Unternehmens- und Führungskonzepten geltend machen. Damit können sie als Bedrohung für Chefärztinnen und -ärzte erlebt werden.

\section{Selbstverständnis variiert zwischen den Professionen}

Uns ist kein anderer Beruf als der des Mediziners bekannt, der seine Aufstiegswege so strikt stufenweise organisiert hat. Nach bestandenem Studienabschluss und langjähriger Lehrzeit als Assistent/in sind die über 30-jährigen Fachärztinnen und -ärzte gut eingespielt im «Leiterlispiel» zwischen Chefärzten, Leitenden Ärzten, Oberärztinnen und Assistenten und haben Heimat gefunden in einer Profession mit starker Eigendefinition. Diese Sozialisation erschwert die Umsetzung der «(Dienstleistungs-)Qualität aus einer Hand» über Berufsgrenzen hinweg. Auch wenn alle unter demselben Dach tätig sind, ist der geteilte Boden in der Haltung keineswegs selbstverständlich, sondern setzt kontinuierliche Absprachen und eine

\section{Abbildung 1}

Das Zusammenspiel der Kräfte.

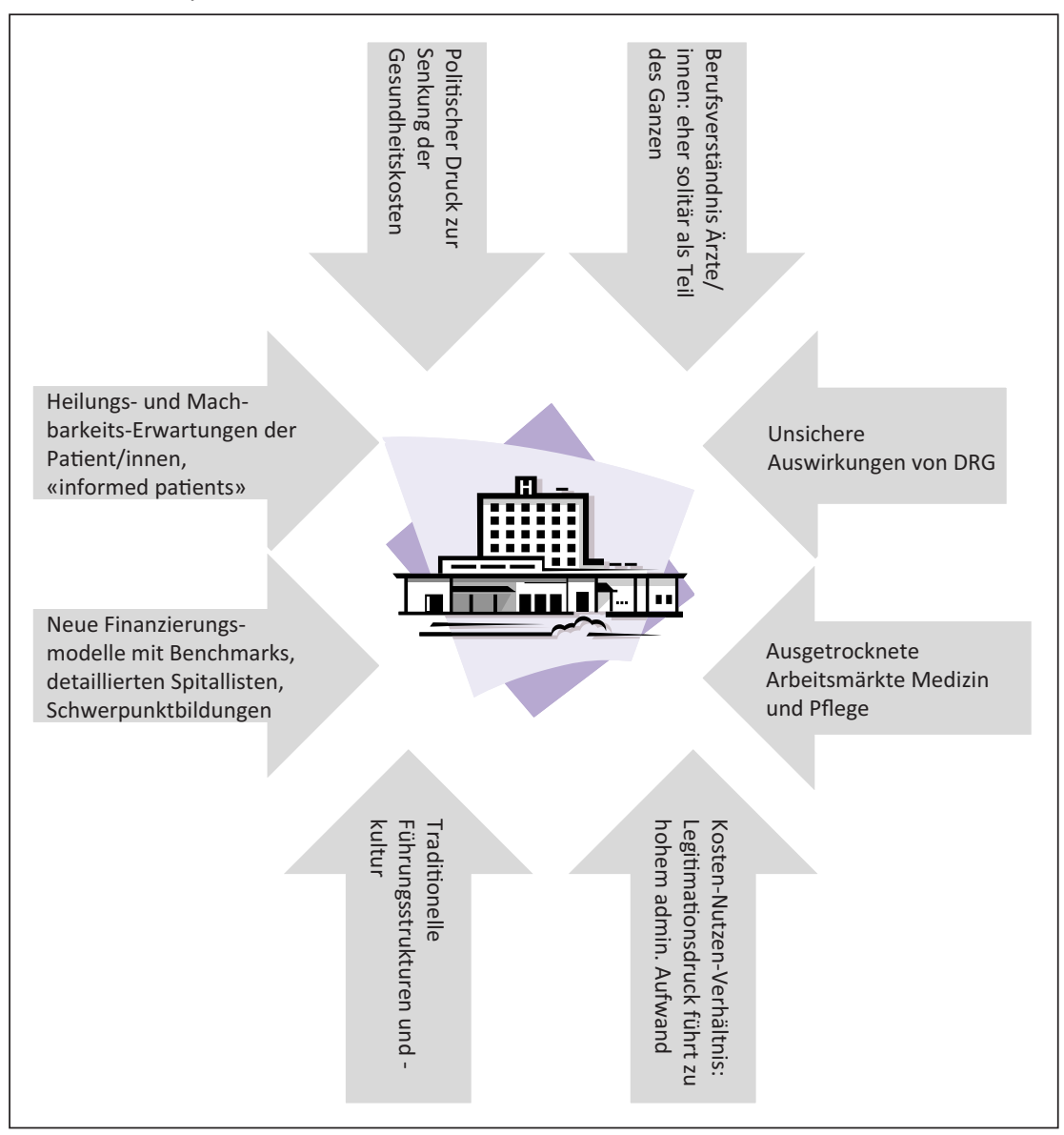

bewusst gepflegte interprofessionelle Kommunikation voraus. Für den Patienten, die Patientin sollte dies spürbar werden in einem Gefühl, rundum gut aufgehoben zu sein, weil «die wirklich miteinander reden». Individualisierte Behandlungspläne sind heute eines der zunehmend vertrauten Instrumente, um diesen kontinuierlichen Dialog im Spitalalltag zu implementieren. Der Weg bis zum umfassenden Dienstleistungsverständnis ist aber noch weit.

Ein kritischer Blick auf die heute gebräuchliche Formulierung der medizinischen Ethik (Genfer Deklaration von 1948) macht demgegenüber deutlich, wie immer noch einzelne Heldinnen und Helden am Werk sind, allenfalls noch ihre «Brüder und Schwestern», eine im professionellen Zusammenhang unserer Meinung nach schlecht passende tribale Metapher. Ein Hinweis auf vertrauensvolle Zusammenarbeit mit anderen Berufsgruppen findet sich hingegen in diesem Text nicht, der immerhin als professionelle Verfassung dient.

\section{Mediziner/innen müssen - fast - alles können}

Nicht alle, die ein Medizinstudium erfolgreich abschliessen, wollen und werden eine Chefarzt-Position erlangen. Dennoch geben die Verhältnisse an der Karrierespitze eines Berufs Auskunft über das Selbstverständnis der gesamten Gruppe.

Wer eine chefärztliche Position erlangen will, muss vieles können: Die Exzellenz in Diagnose und Behandlung ist Grundbedingung für die Erfüllung des Kernauftrages. Betriebswirtschaftliches Wissen ist erforderlich hinsichtlich der Verantwortung für das Budget. Kompetenz in der (Personal-)Führung wiederum ist Voraussetzung, um den Gesamtprozess der Leistungserstellung optimal $\mathrm{zu}$ gestalten, und stellt Anforderungen an die kommunikativen Fähigkeiten, speziell wenn einzelne unterstellte Fachkolleginnen und -kollegen nicht den vom Haus gestellten Erwartungen entsprechen. Im universitären Umfeld kommen Lehre und Forschung als Aufgabe dazu, sowie die Vertretung des eigenen Hauses im Wettbewerb von Publikationen und Kongressen. In der Zusammenarbeit insbesondere mit den pflegerischen Führungspersonen ist Kommunikation auf Augenhöhe mit einer eigenständig sozialisierten und ausgebildeten Berufsgruppe unabdingbar.

So haben wir es mit einem fast übermenschlichen Profil für eine Führungspersönlichkeit zu tun: top im Fachlichen, vertieft in der Forschung, ehrgeizig im internationalen Wettbewerb, kompetent in betriebswirtschaftlichen Fragen, zugewandt und konfliktfähig gegenüber den eigenen Mitarbeitenden, fähig zur interprofessionellen Zusammenarbeit und vorbildhaft als Lehrer oder Lehrerin. Und das alles unter steigendem Kostendruck und zunehmender Kritik an der Ärzteschaft und dem Gesundheitswesen überhaupt. Wir wagen deshalb die Hypothese: Wer sich dieser herkulischen Anforderung stellt, er- 
wartet im Gegenzug Autonomie, Sichtbarkeit und Renommée. Und nicht Unterstellung unter ein betriebswirtschaftlich definiertes und in erster Linie der Gesamtleistung verpflichtetes Führungskonzept.

\section{Ansätze für zukunftstaugliche Lösungen}

Nach wie vor steht die ärztliche Leistung im Zentrum von Diagnose und Behandlung hinsichtlich der Reputation eines Hauses. Somit müssen die Bedürfnisse dieser Berufsgruppe in der Gestaltung der Unternehmenskultur und -strategie sehr ernst genommen werden. Ein Ansatz ist der nüchterne Blick auf durchschnittliche Begabungs- und Belastungsprofile für die Gestaltung von Führungspositionen, sodass aus Übermenschen Kollegen «wie du und ich» werden dürfen. Längerfristig interessante Modelle laufen z.B. unter dem Begriff beyond budgeting. Sie betonen gewährte Gestaltungsräume für alle Positionen mit der damit verbundenen Verantwortung. Kurzfristig können, bei stringenter und entschiedener Führung, auch leichter umsetzbare Massnahmen ausreichen, um die Entwicklung in die skizzierte Richtung voranzutreiben:

- Parallel-Hierarchien mit Aufstiegsmöglichkeiten auf der fachlichen wie auf der ManagementSpur, die es auch ausgewiesenen Fachspezialistinnen und -spezialisten ermöglicht aufzusteigen, ohne dass sie ihnen nicht entsprechende Führungsaufgaben übernehmen müssen. Dieser Ansatz berücksichtigt Fähigkeiten und Neigungen und verlangt dafür Einsicht in die Grenzen der eigenen Einflussmöglichkeiten.

- Echte ärztliche und pflegerische Ko-Leitungen mit der entsprechenden Allokation von Entscheidungskompetenzen. Dieser Ansatz betont

Abbildung 2

Übersicht der Druckfaktoren.

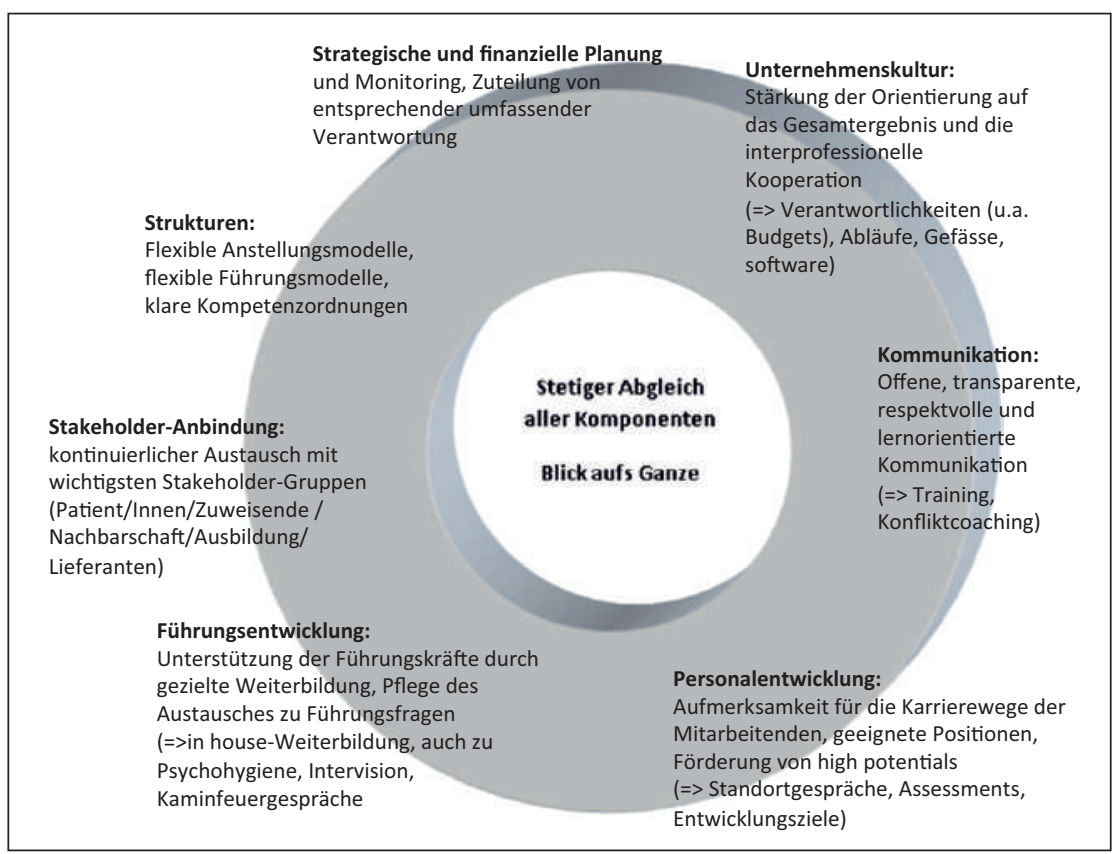

die Interprofessionalität der gesamten Dienstleistung.

- Generell Einführung von Modellen teilzeitlicher Führung zur Stärkung von Karrieren auch während der Familienphase sowie der Geschlechterdurchmischung.

Alle diese Vorschläge bedingen ein hohes Bewusstsein für Interdependenzen sowie für die Notwendigkeit, sorgfältig und präzis zu kommunizieren, auch mit den anderen Berufsgruppen.

- Einführung einer strategischen Personalentwicklung, sodass der ganze Personalzyklus von der Rekrutierung bis zur Pensionierung auf optimalen Einsatz von richtig ausgewählten, geforderten wie geförderten Mitarbeitenden ausgerichtet ist.

- Anstrengungen für Aufbau und Pflege einer professionellen Kommunikation mit den dazugehörenden Grundwerten. Eindämmung von Mikropolitik und intransparentem Gossip, Stärkung des gemeinsamen Lernens, Pflege von Respekt und Verantwortungsübernahme, Belohnung von gemeinsam erreichten Erfolgen.

- Unternehmensweite Führungsweiterbildung mit Austauschgefässen für Führungskräfte aller Professionen im Sinne der gegenseitigen Unterstützung bei anspruchsvollen Führungsfragen.

- Aufmerksamkeit für die persönlichen Belastungen angesichts der anspruchsvollen Aufgaben und der schnellen Veränderungen im Umfeld. Verpflichtende Angebote zur Persönlichkeitsstärkung und Psychohygiene. Ziele: offener Austausch über Überforderungssituationen, attraktive Situation für leistungsbereite Mitarbeitende trotz hoher Belastungen.

- Aufbau einer sachbezogenen, offenen Kommunikation mit externen Stakeholders wie Zuweiser/innen, Patienten/-innen und deren Organisationen, Politik/Finanzierer, Medien, Versicherer, Lieferfirmen, Standort-Gemeinden mit ihren nachbarschaftlichen Kontakten usw. Ziel ist ein gut verankertes Bewusstsein für die Abhängigkeiten auch über die Unternehmensgrenze hinaus, und eine Praxis des Lernens aufgrund regelmässiger Austausch-Gelegenheiten.

\section{Die besondere Rolle der Führungsspitze}

Alle diese Vorschläge müssen, sollen sie ihr Potential entfalten können, von den Mitgliedern der Spitaldirektion nach innen wie nach aussen «verkörpert» und auf alle ihre Implikationen hin bedacht werden. Der Absender solcher strategischer Vorhaben wird von allen Stakeholders scharf beobachtet und muss glaubhaft vorleben, was von anderen verlangt wird, nicht zuletzt in der Zusammenarbeit der Spitaldirektion selber. 